\title{
Analyzing images in frequency domain to estimate the quality of wood particles in OSB production
}

\author{
Ruggero Donida Labati, Angelo Genovese, Enrique Muñoz, Vincenzo Piuri, Fabio Scotti, Gianluca Sforza \\ Department of Computer Science, Università degli Studi di Milano, Italy. \\ Email: \{ruggero.donida, angelo.genovese, enrique.munoz, vincenzo.piuri, fabio.scotti, gianluca.sforza\}@unimi.it
}

\begin{abstract}
The analysis of the quality of particulate materials is of great importance for a variety of research and industrial applications. Most image-based methods rely on the segmentation of the image to measure the particles and aggregate their characteristics. However, the segmentation of particulate materials can be severely affected when the setup is not controlled. For instance, when there are device errors, changes in the light conditions, or when the camera gets dirty because of the dust or a similar substance. All of these circumstances are common in industrial setups, like the one studied in this paper. This work presents a framework for quality estimation based on image processing algorithms that avoids segmentation. The considered application scenario is the online quality control of the production of Oriented Strand Boards (OSB), a type of wood panel frequently used in construction and manufacturing industries. The proposed method quantizes frequency domain into a histogram using a non-parametric method, which is later exploited using computational intelligence to classify the quality of superimposed wood particles deposed on a conveyor belt. The method has been tested using synthetic and real images with different noise conditions. The results illustrate the robustness of the approach and its capability to detect significant quality changes in the wood particles.
\end{abstract}

\section{INTRODUCTION}

Quality control strategies are of paramount importance in industrial production. In particular, automatic control of the productive process allows to drastically reduce the presence of defects in the final product and to constantly monitor the status of the machineries. In productive process of materials composed by small particles (e.g. wood panel production [1], papermaking [2] or mining [3]), control systems are frequently used to analyze the quality of the particles.

In this paper, we consider the production of OSB (Oriented Strand Boards) panels as application scenario. OSBs are a type of wood-based panels widely used in manufacturing and construction industries. To produce them, a mixture of chopped wood particles is glued and pressed. The quality of these wood particles has a direct impact in the appearance, cost, and mechanical properties of the panel [4].

Nowadays, however, online quality analysis is still mainly performed by trained human operators who visually inspect the production line to detect significant changes in the quality of the particles that may affect the characteristics of the final product. The main disadvantage of this process, which is tedious and subjective, is its dependance on the capabilities of the human operator. As a consequence, its accuracy is not standardized. Other approaches are based on the offline measurement of a small amount of material (e.g., using seavers).
To avoid these problems, it is possible to perform an online analysis using vision-based systems. In this way, the analysis is carried out in a fully automatic way and without contact. In an industrial context, the acquisition of images is constrained by the design of production plants, where the materials are usually transported using conveyor belts and free-fall. Thus, images can be acquired placing cameras on top of the conveyor belt or perpendicular to the falling trajectories [5]. This paper focuses on the first setup, analyzing images of the particles deposed on conveyor belts.

In contrast with most common approaches, however, the proposed method does not perform measurements of every particle by using segmentation algorithms. This approach can usually obtain high accuracy, but requires highly controlled environments to obtain satisfactory results. In addition, to analyze the quality of the particles with these techniques, it is necessary to statistically analyze and aggregate the single measures. In particularly critical points of the production, where the environmental conditions are less favorable, it is more useful to use a robust qualitative approach. This paper presents a novel approach that obtains a more robust behavior by analyzing the frequency domain, avoiding segmentation. In addition, the setup can be simplified with respect to methods based on the analysis of single strands, allowing to use a single camera with no particularly high resolution and simple illumination systems. This is particularly useful in the industrial scenario considered in this paper, where it is common to find changes in the environmental conditions that may affect the vision based systems, like: i) changes in light conditions that influence color and gray-tone distributions; ii) dust that can reduce the visibility of the particles that have to be measured; iii) changes in the production materials, for instance the type of wood, which can alter the color of the particles.

The proposed approach, based on the analysis of the frequency domain of the images, has four significant advantages: i) it is flexible against environmental variations, like illumination conditions or dust; ii) the feature extraction is non-parametric; iii) it is fast and robust enough to be used online; iv) and it can detect significant changes in quality during manufacturing. In this way, the capabilities of an expert operator during online production can be emulated, allowing the identification of a deviation from the optimal operating point fixed for the factory.

The remainder of the paper is structured as follows: Section II presents a review of related works, while Section III de- 
scribes the proposed method. Section IV and Section V contain the experimental results and the conclusions, respectively.

\section{RELATED WORK}

Many studies have proposed methods for the analysis of the quality of particulate material using vision systems. The majority of these approaches uses a single camera setup, and a two step algorithm. In the first step, the algorithm segments the image to identify the objects that appear in it, then, in the second step, it evaluates their properties and aggregates them. This approach has been applied to the field of wood panel production, with a framework that joins image processing and computational intelligence to segment and measure woods strands [6]. Furthermore, many approaches have followed this methodology in other fields, such as: biology [7], mining industry [8], [9], [10], or powder classification [11], [12].

However, segmentation is an important source of estimation errors when the setup is not controlled [13]. For instance, when the particles are superimposed, or the illumination is not correct. For this reason, other researchers proposed methods that avoid segmentation. For example, the work in [14] uses a Fourier transform histogram and scale-space decompositions, to roughly estimate particle size distribution. The work in [15] extracts different parameters from the Fourier transform and other mathematical morphological operators and studies their correlation with the quality of the particles. More recently, the study described in [16] employed mathematical morphological opening operations to estimate the quality of aquaculture fish feed pellets, while the work in [17] proposed a general setup based on neural networks, which classifies pixels depending on their probability to belong to a specific granulometric class.

Compared with previous work, the novelty of this paper is threefold: i) it avoids particle segmentation, providing more robustness against environmental variations. ii) it uses a nonparametric method to extract the features that avoids parameter tuning. iii) it is the first approach that thoroughly studies the frequency domain of the images of wood particles, for an online quality analysis of the particles in an industrial setup.

\section{A FRAMEWORK FOR THE QUALITY ANALYSIS OF WOOD STRANDS}

\section{A. Estimating particle quality in wood panels}

The production of OSB panels is carried out by deposing multiple layers of wood particles, or strands, on a conveyor belt. The strands are impregnated with a glue that binds them when pressure and heat are applied [18]. To produce high quality panels, the strands have to fulfill some specified standards. They should be roughly shaped as rectangles and their dimensions have to respect predetermined values. Otherwise, the appearance and mechanical properties of the panel can be damaged, causing severe economic losses. Moreover, panels produced using high quality strands require less glue, and reduce the cost and environmental impact.

In this setup, the estimation of the quality of wood particles using the traditional approach, i.e. trying to segment the strands present in the image, is difficult because:
- the particles have a characteristic shape, with a very long side, that favors the presence of many occlusions, which, in many cases, divide the strands into pieces.

- the color of the particles is, in general, uniform. Thus, it is difficult to differentiate them.

To cope with the aforementioned problems, we introduce a framework for quality estimation based on the analysis of frequency domain. This framework performs three steps: i) image acquisition; ii) analysis of the energy in the frequency domain; iii) neural classification. For the first step, we have developed a synthetic image generator that permits to study in detail the results of the framework, and also a real acquisition setup to be deployed in real industrial environments. In the second step, we quantize the Fourier transform into ring bands of one pixel to produce a histogram that summarizes the data contained in it. We chose the Fourier transform because it is a simple technology that has a low computational complexity compared to other transforms, such as the wavelet transform. This characteristic is particularly useful in in-line real time applications, as the one studied in this paper. The obtained histogram is used in the last step to classify the quality of the wood strands using neural networks.

\section{B. Generation of realistic synthetic images}

To test the robustness and accuracy of the proposed approach against variations in the materials it is useful to use synthetic images. These images facilitate the control of many parameters such as: the size of the strands and its distribution, the angle in which the strands are laid and its distribution, and different lighting conditions. Thus, we can perform very exhaustive tests and rigorously analyze the results.

To generate synthetic images, we have developed a method to create a synthetic mattress. The method randomly distributes wood strands, simulated as rectangles, on a 3-D environment, and then enriches the image with synthetic shadows. Figure 1 shows an example of the obtained mattress from different angles and after the simulation of the shadows.

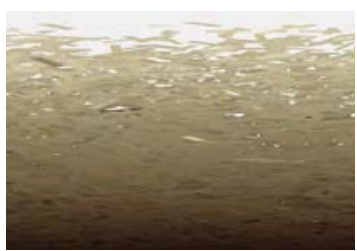

(a)

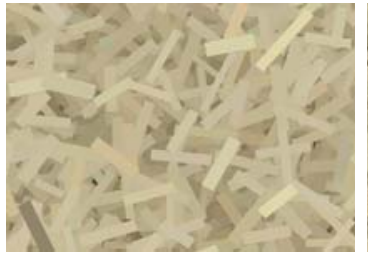

(c)

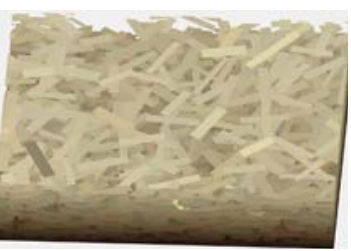

(b)

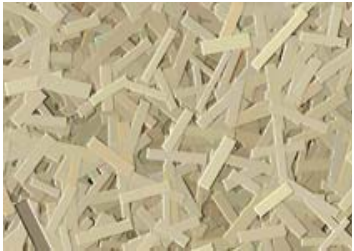

(d)
Fig. 1. A synthetic mattress from different angle. a) side view; b) rotated view; c) top view; d) top view after simulating the shadows. 


\section{Acquisition of real strand images}

To acquire images from the real industrial environment we designed the acquisition setup illustrated in Figure 2. A single camera placed perpendicularly to the stream of wood strands acquires images from the strands laid on the conveyor belt. The illumination system uses two led lights to enhance the visual separation between strands, so that the edges are more visible in the images. This setup has the advantage of requiring limited hardware costs. In addition, it permits to perform a fast analysis of the acquired data.

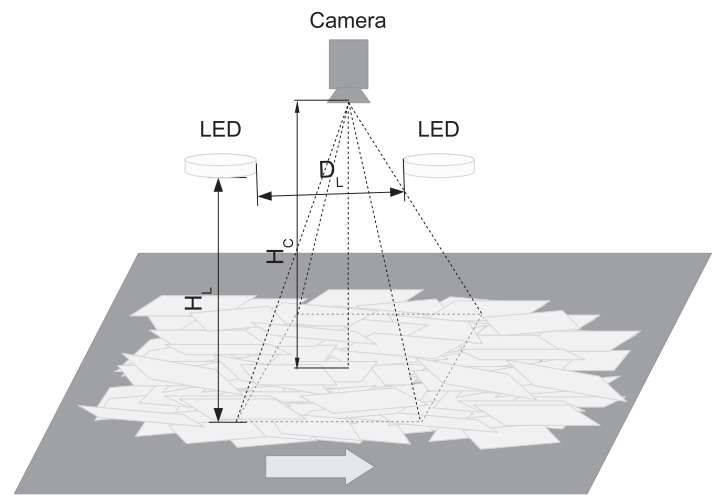

Fig. 2. Schema of the used acquisition setup.

\section{Analysis of the energy in the frequency domain}

The proposed method uses the 2-D Discrete Fourier Transform of an image, defined as [19]:

$$
F(u, v)=\sum_{x=0}^{M-1} \sum_{y=0}^{N-1} f(x, y) e^{-j 2 \pi\left(\frac{u x}{M}+\frac{v y}{N}\right)}
$$

for frequencies $u=0,1, \cdots, M-1$ and $v=0,1, \cdots, N-1$, where $f(x, y)$ denotes an $M \times N$ image. In general $F(u, v)$ is complex, and can be analyzed in terms of magnitude and phase. Let $R(u, v)$ and $I(u, v)$ be, respectively, the real and imaginary part of $F(u, v)$. The proposed method analyzes the power spectrum of the transform, defined as:

$$
P(u, v)=R^{2}(u, v)+I^{2}(u, v)
$$

In particular, $P(u, v)$ is partitioned into radial bands of one pixel of width. Being each band represented by the set

$$
B(r)=\left\{(u, v): r=\left\lfloor\sqrt{\left(u-u_{c}\right)^{2}+\left(v-v_{c}\right)^{2}}\right\rfloor\right\}
$$

for $r=1, \cdots, R$, where $u_{c}$ and $v_{c}$ are the coordinates of the

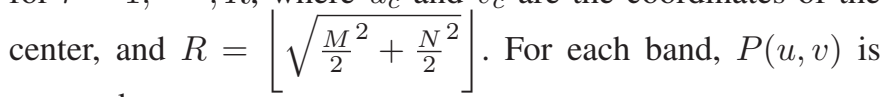
summed

$$
H(r)=\sum_{(u, v) \in B(r)} P(u, v)
$$

Then each $H(r)$ is normalized:

$$
N(r)=H(r) / \sum_{o=1}^{R} H(o)
$$

Each $N(r)$ represents the energy present in frequency $r$, both in vertical and horizontal direction. Bands near the center represent low frequencies (or large wavelengths) and those away from the center high frequencies.

The final result is an energy histogram that summarizes the energy contained in each frequency present in the image. This histogram is a useful method to study the texture of an image. The shape of the histogram changes depending on the quality of the strands present in the image. For low quality strands, the histogram accumulates more energy in high frequencies, while for high quality strands, the energy is higher in lower frequencies. Fig. 3 illustrates histogram calculation for real images of wood strands, showing the images, their Fourier transform and the histograms.

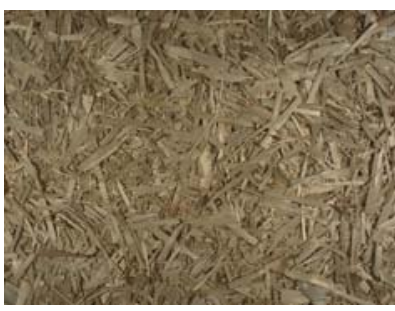

(a)

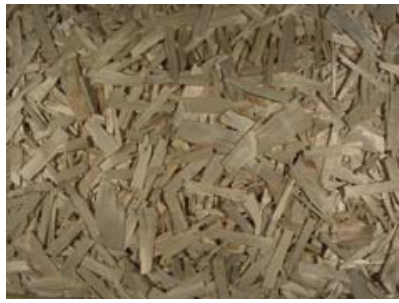

(c)

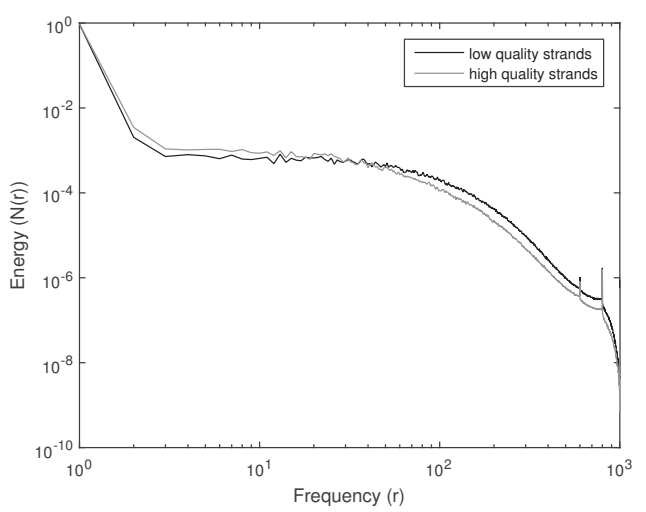

(e)

Fig. 3. Example of the histograms obtained from images with resolution $1200 \times 1600$. a) image including real strands of low quality; b) magnitude of the Fourier transform for strands of low quality; c) image including real strands of high quality; d) magnitude of the Fourier transform for strands of high quality; e) comparison of the two energy histograms as a log-log plot.

\section{E. Neural classification of strand quality}

To determine the quality of the strands present in the image, we use neural network classifiers. These classifiers exploit the information contained in the energy histogram. In particular 


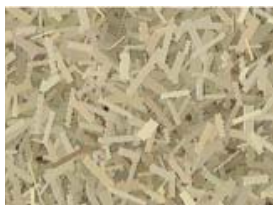

(a)

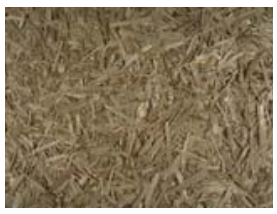

(f)

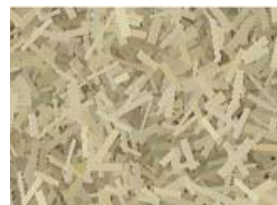

(b)

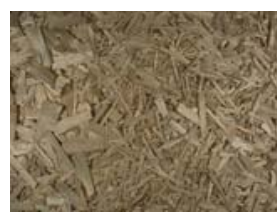

(g)

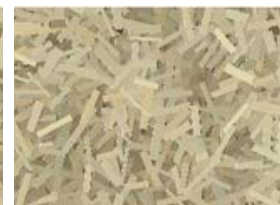

(c)

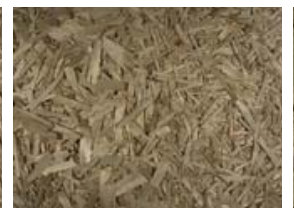

(h)

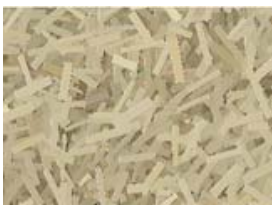

(d)

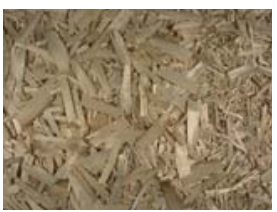

(i)

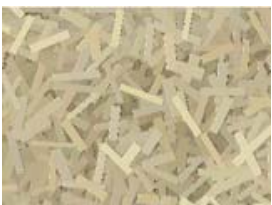

(e)

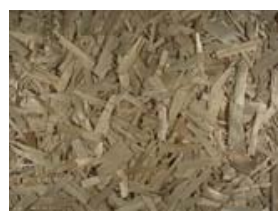

(j)

Fig. 4. (a-e) Examples of the images in the database of synthetic images. (f-j) Examples of the images in the database of real images. The size of the strands present in the images increases from left to right.

the energy histogram contains $R$ bins that can be directly used as features for classification. However, for large images, $R$ can be very high, which makes more difficult the training of the neural network. For this reason, we propose a set of features that summarize the information contained in the $R$ bins.

These features are general features used to describe histograms, and, for instance, have been previously used to describe the histogram of intensity levels of an image [19]. The features include:

- Mean: $\left(F_{M}\right)$ : a measure of the average energy computed as: $F_{M}=\sum_{r=1}^{R} N(r) r$.

- Standard deviation $\left(F_{S}\right)$ : a measure of the average variability in energy calculated as: $F_{S}=$ $\sqrt{\sum_{r=1}^{R} N(r)\left(r-F_{M}\right)^{2}}$

- Smoothness $\left(F_{S m}\right)$ : a measure of the relative evenness of the histogram computed as: $F_{S m}=1-1 /\left(1+F_{S}^{2}\right)$.

- Third moment $\left(F_{T}\right)$ : this feature measures the skewness of the energy histogram. It is calculated as: $F_{T}=$ $\sum_{r=1}^{R} N(r)\left(r-F_{M}\right)^{3}$.

- Uniformity $\left(F_{U}\right)$ : this features indicates the uniformity of the energy histogram. It is computed as: $F_{U}=$ $\sum_{r=1}^{R} N^{2}(r)$.

- Entropy $\left(F_{E}\right)$ : a measure of the randomness in the energy histogram. Estimated as $F_{E}=-\sum_{r=1}^{R} N(r) \log _{2} N(r)$.

- Kurtosis $\left(F_{K}\right)$ : an estimation of whether the energy histogram is peaked or flat relative to a normal distribution. It is computes as: $F_{K}=\sum_{r=1}^{R} N(r)\left(r-F_{M}\right)^{4}$.

\section{EXPERIMENTAL RESULTS}

\section{A. Test database of synthetic images}

To test the accuracy and robustness of the proposed approach against slight variations in the quality of the materials we created a database of 500 synthetic images. These images had a resolution of $1600 \times 1200$ pixels and we divided them into five quality classes, $C_{1}, \cdots, C_{5}$. We have created each quality class using different normal distributions for the sizes of the strands. The normal distributions to generate the length of the strands used the parameters $\mu=\{60,70,80,90,100\}$ and $\sigma=\{40,35,30,25,20\}$, while, to generate the width of the strands we used the parameters $\mu=\{15,16.25,17.5,18.75,20\}$ and $\sigma=\{4,4.5,5,5.5,6\}$. These parameters correspond to the measures in millimeters of strands in the real setup. We created 100 images for each quality class. Figure 4 shows examples of the different classes.

To test the behavior of the proposed approach in a scenario where the environmental conditions are not ideal, we extended the database by adding noise to the images, obtaining a final database with 4500 images. We studied two kinds of noise (See Figure 5 for examples of the noise added to the images):

- device noise, simulated as salt and pepper noise with different densities $d=\{0.01,0.02,0.04,0.08\}$;

- noise caused by a dirty lens, simulated as Gaussian blur with different kernel sizes $\{2,4,8,16\}$ and standard deviations $\{1,2,4,8\}$.

\section{B. Test database of real images}

To check the accuracy of the system in a real scenario, we obtained a set of images of real strands using the setup in Figure 2. We used a commercial color CCD camera and two white LED lights. The configuration of the setup was: distance between the LEDs $D_{L}=1300 \mathrm{~mm}$, distance between the lights and the mattress $H_{L}=1100 \mathrm{~mm}$, and distance between the camera and the mattress $H_{C}=810 \mathrm{~mm}$. The parameters of the camera were: focal length $f=8 \mathrm{~mm}$, shutter time $t_{s}=1 / 58 \mathrm{~s}$, and gain $G=0.00 \mathrm{~dB}$, chosen in order to minimize noise or motion blur. The size of the captured images is $W \times H=1600 \times 1200$ pixels and describe an area with size $W \times H \approx 740 \times 560 \mathrm{~mm}$.

The database of real images contained 50 images, divided into five quality classes, $C R_{1}, \cdots, C R_{5}$. Each class contained a different proportion of high and low quality particles, with approximate proportions of low quality particles of $\{1,0.7,0.5,0.3,0\}$ starting from $C R_{1}$. We captured 10 different images for each $C R_{i}$. Figure 4 shows examples of the different classes. As in the case of the synthetic database, the database was extended by adding noise to the real images, 
obtaining a final database with 450 images. Figure 5 shows examples of the noise added to the images.

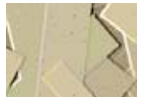

(a)

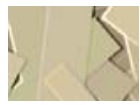

(e)

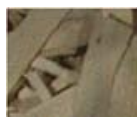

(i)

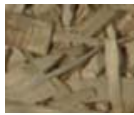

(m)

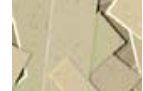

(b)

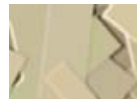

(f)

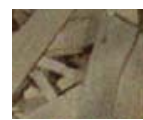

(j)

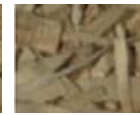

(n)

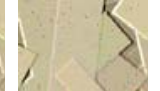

(c)

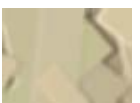

(g)

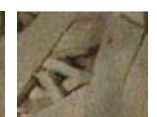

(k)

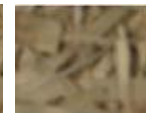

(o)

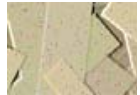

(d)

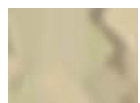

(h)

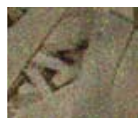

(1)

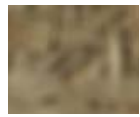

(p)
Fig. 5. Examples of different quantity of salt and pepper noise in synthetic (a-d) and real images (i-l) and Gaussian blur in synthetic (e-h) and real images (m-p).

\section{Experimental procedure}

For each image in the database, we used the proposed method to estimate the quality of the strands. In particular, we used a Feed Forward Neural Network with one input layer, one hidden layer and one output layer. The input layer used 7 nodes, the number of input features, and the output layer used 5 nodes, with a linear transfer function. We tested different number of nodes, with tan-sigmoidal transfer functions, in the hidden layer: 10,15,20,50,100. A resilient back-propagation algorithm, using 50 epochs, was used for the training. We considered only neural networks with a single hidden layer, since they are universal approximators. As figure of merit we considered the accuracy $=\frac{\mid \text { True positives }|+| \text { True negatives } \mid}{\mid \text { Test database } \mid}$, where $|\cdot|$ indicates the cardinality of a set. The accuracy was averaged using 10-fold cross validation.

\section{Analysis of the feature set}

As a first test we used the synthetic database to study the performance of different sets of features. We compared the results obtained using the following sets of features:

- the raw energy histogram $\left(S_{H}\right)$.

- the set of features proposed in the paper: $\left\{F_{M}, F_{S}, F_{S m}\right.$, $F_{T}, F_{U}, F_{E}$ and $\left.F_{K}\right\}\left(S_{S}\right)$.

- the set of textural features based on the Gray-level cooccurrence matrix $\left(S_{C}\right)$ [20]. This set contains 4 features: homogeneity, energy, correlation and contrast.

- the set of textural features based on the analysis of the histogram of intensity levels $\left(S_{I}\right)$ [19]. This set contains 6 features: mean, standard deviation, smoothness, third moment, uniformity and entropy.
TABLE I

RESULTS OF THE NEURAL CLASSIFICATION USING DIFFERENT FEATURE SETS.

\begin{tabular}{ccccc} 
& $S_{H}$ & $S_{S}$ & $S_{C}$ & $S_{I}$ \\
\cline { 2 - 5 } accuracy & $84.2 \%$ & $94.3 \%$ & $93.4 \%$ & $93.8 \%$
\end{tabular}

Note: $S_{H}=$ raw energy histogram; the proposed approach $S_{S}=F_{M}, F_{S}, F_{S m}, F_{T}$, $F_{U}, F_{E}, F_{K} ; S_{C}=$ Gray-level co-occurrence matrix features [20]; $S_{I}=$ histogram of intensity levels features [19].

TABLE II

RESULTS OF THE CLASSIFICATION USING DIFFERENT LEARNING TECHNIQUES.

\begin{tabular}{ccccc} 
& $\mathrm{kNN}-1$ & $\mathrm{kNN}-3$ & Tree & FFNN-15 \\
\cline { 2 - 5 } accuracy & $89.4 \%$ & $93.4 \%$ & $93.2 \%$ & $94.3 \%$
\end{tabular}

Note: FFNN-15 = Feed Forward Neural Network with 15 neurons in the hidden layer.

The obtained results are shown in Table I. These results illustrate the improvement obtained by summarizing the information contained on the energy histogram, as opposed to directly use the full histogram. In addition, the results indicate the suitability of the proposed features compared with other features commonly used in the literature.

\section{E. Analysis of the performance of the neural model}

This section analyzes the performance of the neural network with the synthetic images. We compared the results obtained by the proposed neural classifier and other wellknown techniques, k-Nearest Neighbor and tree classifiers. Table II presents the obtained results.

The obtained results indicate that the neural classifier outperformed the other methods on the considered dataset. In fact, the neural network obtained a significant reduction of the classification error. However, the analysis of which computational intelligence method obtains the best results is out of the scope of this work, since it also depends on the specific data of the studied application. Other computational intelligence techniques, like Support Vector Machines, could be analyzed in future research studies.

\section{F. Analysis of the accuracy in noisy environments}

Table III presents the results obtained by the classifier for synthetic images containing different levels of salt and pepper noise. The results illustrate the robustness of the approach against this kind of noise, with accuracy values over $90 \%$ even for high noise densities.

Table III shows the accuracy obtained for synthetic images containing different levels of Gaussian blur. The obtained results demonstrate that the framework can deal Gaussian blur without suffering significant accuracy drops. In particular, the obtained accuracy values remain around $90 \%$ except for the highest noise, for which, nonetheless, the accuracy is still high.

\section{G. Analysis of the performance with real images}

This section analyzes the results obtained for the images captured from a real mattress, both with and without noise. Table IV presents the obtained results. These results illustrate 
TABLE III

RESULTS OBTAINED FOR SYNTHETIC IMAGES WITH NOISE

\begin{tabular}{ccccc} 
& \multicolumn{4}{c}{ salt and pepper density } \\
\cline { 2 - 5 } & 0,01 & 0,02 & 0,04 & 0,08 \\
\cline { 2 - 5 } accuracy & $94.2 \%$ & $94.0 \%$ & $92.8 \%$ & $93.6 \%$
\end{tabular}

Gaussian kernel (pixels) std dev

\begin{tabular}{ccccc}
\cline { 2 - 5 } accuracy & $2_{1}$ & $4_{2}$ & $8_{4}$ & $16_{8}$ \\
\cline { 2 - 5 } & $93.8 \%$ & $93.5 \%$ & $91.5 \%$ & $88.1 \%$
\end{tabular}

TABLE IV

RESULTS OBTAINED FOR REAL IMAGES

\begin{tabular}{cccccc} 
& \multicolumn{5}{c}{ salt and pepper density } \\
\cline { 2 - 6 } & no-noise & 0,01 & 0,02 & 0,04 & 0,08 \\
\cline { 2 - 6 } accuracy & $91.6 \%$ & $90.0 \%$ & $89.4 \%$ & $88.2 \%$ & $90.4 \%$
\end{tabular}

Gaussian kernel (pixels) std dev

\begin{tabular}{cccccc} 
& no-noise & $2_{1}$ & $4_{2}$ & $8_{4}$ & $16_{8}$ \\
\cline { 2 - 6 } accuracy & $91.6 \%$ & $94.4 \%$ & $96.6 \%$ & $96.4 \%$ & $92.2 \%$
\end{tabular}

that the proposed approach is also suitable to predict the quality of the strands in real industrial scenarios, even in a noisy environment.

\section{CONCLUSION}

This work presents a framework for the estimation of particle quality in the industrial area of OSB production. The proposed framework uses an innovative approach that avoids segmentation, which represents the most important source of estimation errors when the setup is not controlled [13]. In particular, it quantizes the power spectrum of the image in bands of one pixel that summarize the energy contained in each frequency. The whole spectrum is considered, without filtering any band. This approach has the advantages of being independent from the zoom, and avoiding the tuning of the filters. Using this information the framework creates a set of non-parametric features exploited by a neural model that permits to predict the quality of the particles present in an image. Several tests have been applied to analyze the performance of the approach under different circumstances, demonstrating its capability to detect changes in the quality of wood particles, even under noisy conditions.

As future work we plan to apply evolutionary computation to perform band selection/weighting. This process will optimize the differentiation between the quality classes while maintaining a high adaptability.

\section{ACKNOWLEDGMENT}

This work was supported in part by the EC within the 7FP under grant agreement 308630 (I-PAN).

\section{REFERENCES}

[1] F. Ding, M. Benaoudia, P. Bédard, R. Lanouette, C. Lejeune, and P. Gagné, "Wood chip physical quality definition and measurement," Pulp and Paper Laboratory, Centre de recherche industrielle du Qubec (CRIQ), Quebec, Canada, Tech. Rep, 2005.
[2] M. Lopez, J. Vilan, J. Matias, and J. Taboada, "Quality control of woodpulp chips using a 3d laser scanner and functional pattern recognition," in Proc. of the IEEE Int. Symp. on Industrial Electronics, 2007. ISIE 2007, 2007, pp. 1773-1778.

[3] M. J. Thurley, "Automated online measurement of limestone particle size distributions using 3d range data," Journal of Process Control, vol. 21, no. 2, pp. 254-262, 2011.

[4] P. Camorani, M. Badiali, D. Francomacaro, M. Gamassi, V. Piuri, F. Scotti, and M. Zanasi, "A classification method for wood types using fluorescence spectra," in Proc. of the IEEE Instrumentation and Measurement Technology Conference, 2008. IMTC 2008, 2008, pp. 1312-1315.

[5] R. Donida Labati, A. Genovese, V. Piuri, and F. Scotti, "A virtual environment for the simulation of $3 \mathrm{~d}$ wood strands in multiple view systems for the particle size measurements," in Proc. of the 2013 IEEE Int. Conf. on Computational Intelligence and Virtual Environments for Measurement Systems and Applications (CIVEMSA), 2013, pp. 162-167.

[6] R. Donida Labati, A. Genovese, E. Muñoz, V. Piuri, F. Scotti, and G. Sforza, "Improving osb wood panel production by vision-based systems for granulometric estimation," in Proc. of the 1st Int. Forum on Research and Technologies for Society and Industry - Leveraging a better tomorrow (RTSI 2015), Turin, Italy, 2015, pp. 557-562.

[7] V. Zapater, L. Martnez-Costa, and G. Ayala, "A granulometric analysis of specular microscopy images of human corneal endothelia," Computer Vision and Image Understanding, vol. 97, no. 3, pp. 297-314, 2005

[8] K. C. Williams, W. Chen, S. Weeger, and T. J. Donohue, "Particle shape characterisation and its application to discrete element modelling," Particuology, vol. 12, pp. 80-89, 2014.

[9] M. Salehizadeh and M. Sadeghi, "Size distribution estimation of stone fragments via digital image processing," Advances in Visual Computing, pp. 329-338, 2010.

[10] T. K. Koh, N. Miles, S. Morgan, and B. Hayes-Gill, "Image segmentation of overlapping particles in automatic size analysis using multi-flash imaging," in Proc. of the IEEE Workshop on Applications of Computer Vision, 2007. WACV'07. IEEE, 2007, pp. 47-47.

[11] A. Boschetto and V. Giordano, "Powder sampling and characterization by digital image analysis," Measurement, vol. 45, no. 5, pp. 1023-1038, 2012.

[12] A. Mazzoli and O. Favoni, "Particle size, size distribution and morphological evaluation of airborne dust particles of diverse woods by scanning electron microscopy and image processing program," Powder Technology, vol. 225, pp. 65-71, 2012.

[13] S. Ferrari, V. Piuri, and F. Scotti, "Image processing for granulometry analysis via neural networks," in Proc. of the 2008 IEEE Int. Conf. on Computational Intelligence for Measurement Systems and Applications (CIMSA 2008), 2008, pp. 28 -32.

[14] A. Zadorozny, H. Zhang, and M. Jagersand, "Granulometry using image transformation techniques," in Proc. of the International Conference on Vision Interface, 2002, pp. 433-438.

[15] A. Ledda, J. Quintelier, P. Samyn, P. De Baets, and W. Philips, "Quantitative image analysis with mathematical morphology," in Proc. of ProRISC, vol. 2003, 2003, pp. 399-406.

[16] M. G. Ljungqvist, M. E. Nielsen, B. K. Ersbll, and S. Frosch, "Image analysis of pellet size for a control system in industrial feed production," PLoS ONE, vol. 6, no. 10, p. e26492, 2011.

[17] S. Ferrari, V. Piuri, and F. Scotti, "Virtual environment for granulometry analysis," in Proc. of the IEEE Int. Conf. on Virtual Environments, Human-Computer Interfaces and Measurement Systems (VECIMS 2008), 2008, pp. 156-161.

[18] H. Thoemen, M. Irle, and M. Sernek, Wood-based panels : an introduction for specialists. Brunel University Press, 2010.

[19] R. C. Gonzalez, Digital image processing, 2nd ed. Upper Saddle River, N.J: Prentice Hall, 2002.

[20] R. M. Haralick, K. Shanmugam, and I. H. Dinstein, "Textural features for image classification," IEEE Trans. on Systems, Man and Cybernetics, no. 6, pp. 610-621, 1973. 